\title{
Malignant Labia Majora Neoplasm
}

National Cancer Institute

\section{Source}

National Cancer Institute. Malignant Labia Majora Neoplasm. NCI Thesaurus. Code

C7638.

A malignant neoplasm that affects the labia majora. 